\title{
The mechanical advantage of the magnetosphere: solar-wind-related forces in the magnetosphere-ionosphere-Earth system
}

\author{
V. M. Vasyliūnas \\ Max-Planck-Institut für Sonnensystemforschung, 37191 Katlenburg-Lindau, Germany
}

Received: 20 November 2006 - Accepted: 22 January 2007 - Published: 1 February 2007

\begin{abstract}
Magnetosphere-ionosphere interactions involve electric currents that circulate between the two regions; the associated Lorentz forces, existing in both regions as matched opposite pairs, are generally viewed as the primary mechanism by which linear momentum, derived ultimately from solar wind flow, is transferred from the magnetosphere to the ionosphere, where it is further transferred by collisions to the neutral atmosphere. For a given total amount of current, however, the total force is proportional to $\mathcal{L} B$ and in general, since $\mathcal{L}^{2} B \sim$ constant by flux conservation, is much larger in the ionosphere than in the magnetosphere ( $\mathcal{L}=$ effective length, $B=$ magnetic field). The magnetosphere may be described as possesing a mechanical advantage: the Lorentz force in it is coupled with a Lorentz force in the ionosphere that has been amplified by a factor given approximately by the square root of magnetic field magnitude ratio ( $\sim 20$ to 40 on field lines connected to the outer magnetosphere). The linear momentum transferred to the ionosphere (and thence to the atmosphere) as the result of magnetic stresses applied by the magnetosphere can thus be much larger than the momentum supplied by the solar wind through tangential stress. The added linear momentum comes from within the Earth, extracted by the Lorentz force on currents that arise as a consequence of magnetic perturbation fields from the ionosphere (specifically, the shielding currents within the Earth that keep out the time-varying external fields). This implies at once that Fukushima's theorem on the vanishing of ground-level magnetic perturbations cannot be fully applicable, a conclusion confirmed by re-examining the assumptions from which the theorem is derived. To balance the inferred Lorentz force within the Earth's interior, there must exist an antisunward mechanical stress there, only a small part of which is the acceleration of the entire Earth system by the net force exerted on it by the
\end{abstract}

Correspondence to: V. M. Vasyliūnas

(vasyliunas@mps.mpg.de) solar wind. The solar-wind interaction can thus give rise to internal forces, significantly larger than the force exerted by the solar wind itself, between the ionosphere and the neutral atmosphere as well as within the current-carrying regions of the Earth's interior.

Keywords. Ionosphere (Ionosphere-atmosphere interactions) - Magnetospheric physics (Magnetosphereionosphere interactions; Solar wind-magnetosphere interactions)

\section{Introduction}

Transfer of momentum from the solar wind stands second only to transfer of energy and mass in any list of scientific objectives for an investigation of the magnetosphere. The basic concepts were introduced in a classic paper by Siscoe (1966). The solar wind exerts a net antisunward force on the magnetosphere, both through a normal stress (related to deflection of its flow) and through a tangential stress (now ascribed primarily to connection of magnetic fields through the magnetopause). This force is ultimately transferred to the planet Earth, a conclusion argued by Siscoe (1966) on the grounds that nothing else is massive enough to sustain the force without being blown away but also derivable, as I show here (Sect. 5), from a more specific argument. Siscoe (1966) describes the force on the Earth as a magnetic force, exerted by the gradient of the field from magnetopause and magnetotail currents that interacts with the Earth's dipole moment. At the Earth's surface the gradient appears as the day-night asymmetry of the Chapman-Ferraro disturbance field; from the condition that the gradient should match the solar-wind force on the magnetosphere, Siscoe (1966) infers an upper limit to the asymmetry (noon higher than midnight by no more than $\sim$ a few nT).

Published by Copernicus GmbH on behalf of the European Geosciences Union. 
More recently, attention has increasingly been directed toward the possible contribution of drag forces on the highlatitude ionosphere, associated with the tangential stress of the solar wind and mediated by Birkeland (magneticfield-aligned) currents between the ionosphere and the outer magnetosphere, in particular the so-called region 1 currents (Iijima and Potemra, 1976). Siscoe et al. (2002) propose that, in the limit of very strong solar wind interaction (transpolar potential saturation regime), stresses associated with region 1 currents assume the dominant role in transmitting forces exerted by and on the solar wind.

In all these discussions it appears mostly to have been taken for granted that the linear momentum supplied by the solar wind is simply transferred to the other regions and that any force exerted on the ionosphere or on the Earth is equal to the force applied by the solar wind. The purpose of this paper is to point out that this is not the case: as a consequence of very simple considerations of magnetic field geometry, any given current system flowing between the magnetosphere and the ionosphere implies a much larger Lorentz force in the ionosphere than it does in the magnetosphere. This conclusion has been reached independently by Siscoe and Siebert (2006) for the special case of forces associated with region 1 currents, in the context of a general discussion of solar wind forces on the magnetosphere and their relation to observed thermospheric effects during large magnetic storms.

In effect, as far as Lorentz forces are concerned, the magnetosphere may be said to act on the ionosphere as a simple machine (e.g. lever or pulley) with a very considerable mechanical advantage. A force applied by the solar wind may then appear as a much larger force on the ionosphere, raising the question (since linear momentum is conserved) where the excess linear momentum is coming from. I show that it is coming from Lorentz forces exerted on currents within the Earth's interior that arise as the result of the disturbance fields from the ionosphere; the various arguments commonly made (notably the celebrated theorem of Fukushima, 1969, 1971, 1985b) that these disturbance fields are unimportant below the ionosphere must therefore be of limited applicability.

\section{Mechanical and electromagnetic stress balance equa- tions}

Conservation of linear momentum in an extended medium is discussed in any textbook of fluid mechanics or plasma physics (see, e.g., Landau and Lifshitz, 1959; Rossi and Olbert, 1970; Siscoe, 1983, the latter a treatment intended specifically for magnetospheric applications). The local (differential) form of the momentum equation (written in the standard conservation form: partial time derivative of density of conserved quantity plus divergence of flux density of conserved quantity) is

$\partial \boldsymbol{G} / \partial t+\nabla \cdot(\rho \boldsymbol{V} \boldsymbol{V}+\mathbf{P}-\mathbf{T})=0$ where

$\boldsymbol{G}=\rho \boldsymbol{V}+(\boldsymbol{E} \times \boldsymbol{B} / 4 \pi c)$

is the linear momentum per unit volume, the first term representing the momentum of bulk flow of the medium and the second the momentum of the electromagnetic field (I use Gaussian units throughout); $\rho, \boldsymbol{V}$, and $\mathbf{P}$ are, respectively, the mass density, bulk flow velocity, and pressure tensor of the medium; $\mathbf{T}$ is the sum of the Maxwell stress tensor $\mathbf{T}_{M}$ (the divergence of which is equal to the Lorentz force per unit volume) plus the stress tensors representing any other forces. The global form of the momentum equation, obtained by integrating Eq. (1) over a given volume, states that the total linear momentum contained within the volume changes at a rate that is given by a surface integral over the boundary of the volume; the three terms in the surface integral, corresponding to the three terms within the divergence in Eq. (1), represent the net rate of momentum transfer across the boundary by bulk flow (first term), by thermal motion of particles (second term: pressure tensor), and by the action of forces on the medium (third term: stress tensors of the various forces). That the total force acting on the medium within the volume can be expressed as the surface integral of the appropriate stress tensor is an essential condition for conserving total linear momentum. For an isolated system, with negligible surface terms, the total linear momentum remains constant.

Under the usual assumptions of charge quasi-neutrality of the plasma, nonrelativistic bulk flows, and Alfvén speed $V_{A}{ }^{2} \ll c^{2}$, the electromagnetic contribution to the linear momentum density (second term on the right-hand side of Eq. (2) and the electric-field terms in the Maxwell stress tensor can be neglected. The Lorentz force then reduces to just the magnetic force, given by the divergence of the Maxwell stress tensor

$\boldsymbol{J} \times \boldsymbol{B} / c=\nabla \cdot \mathbf{T}_{M}=\nabla \cdot\left[\boldsymbol{B} \boldsymbol{B} / 4 \pi-\mathbf{I}\left(B^{2} / 8 \pi\right)\right]$

(I is the unit dyad, $\mathbf{I}_{i j}=\delta_{i j}$ ); to derive relation (3), Ampère's law

$\mathbf{J}=(c / 4 \pi) \nabla \times \mathbf{B}$

needs to be invoked.

For the purposes of this paper, three aspects of the above general formulation, embodied in Eqs. (4), (3), and (1), are important:

1) Equation (4) implies the current continuity condition $\boldsymbol{\nabla} \cdot \boldsymbol{J}=0$, which is the basis for the results discussed in Sect. 3 .

2) Equation (1) in its integral form, applied to a sequence of nested boundary surfaces from the magnetopause inward to the center of the Earth, forms the basis for the discussion in Sect. 5 of linear momentum transfer from the solar wind.

3) Equation (3), by allowing the Lorentz force integrated over a given volume to be calculated as the Maxwell stress tensor integrated over the surface bounding the volume, implies a reciprocity relation that is the basis for the limitations 
to Fukushima's theorem discussed in Sect. 6. For any chosen volume, the total Lorentz force exerted on currents within the volume can be calculated in this way, with no constraints from current closure; even if the current does not close within the volume, the total Lorentz force is a well defined physical quantity - in contrast to the magnetic field of an unclosed current segment, which in general has only a mathematical significance (Vasyliūnas, 1999). This force is given, however, by the surface integral of the Maxwell stress tensor, and one may view the surface in two ways: either as the outer boundary of the chosen volume, or as the inner boundary of the rest of space, the difference being only in the sign of the normal vector. Hence the total Lorentz force on currents (closed or unclosed) within any volume is precisely equal and opposite to the total Lorentz force on currents outside that volume; this applies to each Cartesian vector component, taken separately. A corollary is that magnetic fields due entirely to currents confined within a volume exert no net force on the volume.

\section{Relation between Lorentz forces in the magneto- sphere and the ionosphere}

The interaction between the magnetosphere and the ionosphere proceeds to a large extent by means of electric currents that flow in both regions, coupled by Birkeland (magnetic-field-aligned) currents between the two; much of the classical theory of magnetosphere-ionosphere coupling consists simply of the self-consistency conditions on these currents and on the associated plasma flows and stresses. As a prototypical example, Fig. 1 shows a sketch of the complete system of region 1 currents. The segment $\mathcal{I}-\mathcal{I}^{\prime}$ flows from dawn to dusk in the polar ionosphere. The segment $\mathcal{M}^{\prime}-\mathcal{M}$ flows from dusk to dawn somewhere in the outer magnetosphere; the precise location has not been conclusively identified but is generally assumed to be near the magnetopause, its boundary layers, or the adjacent magnetosheath. The segments $\mathcal{M}-\mathcal{I}$ and $\mathcal{I}^{\prime}-\mathcal{M}^{\prime}$ are the region 1 currents proper: Birkeland currents flowing into the ionosphere on the dawn side and out of the ionosphere on the dusk side.

Current continuity imposes a relation between the currents in the different segments. With the segments idealized, for simplicity, as line currents in Fig. 1 (in reality, of course, they have appreciable width in the transverse directions), the relation is simply that the total current $I$ is the same everywhere. The total Lorentz force exerted on the plasma in each segment can now be computed. The segments $\mathcal{M}-\mathcal{I}$ and $\mathcal{I}^{\prime}-\mathcal{M}^{\prime}$ are (very nearly) aligned with the ambient magnetic field and hence have negligible Lorentz force. At the ionospheric segment $\mathcal{I}-\mathcal{I}^{\prime}$, the ambient magnetic field $B_{i}$ is downward, and the Lorentz force $F_{i}$ is directed antisunward. At the magnetospheric segment $\mathcal{M}-\mathcal{M}^{\prime}$, the vertical component of the ambient magnetic field $B_{m}$ is (for the assumed location) likewise downward but, with the current reversed,

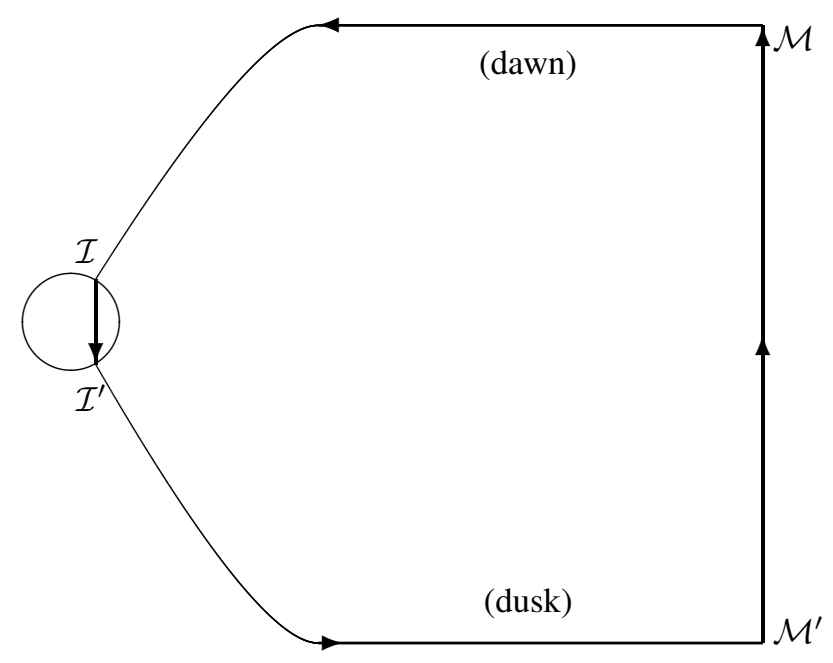

Fig. 1. Schematic diagram of region 1 currents in Northern Hemisphere, viewed from above the north pole (Sun to the left).

the Lorentz force $F_{m}$ in the plane is directed sunward. The magnitude of the forces is

$F_{i} \approx(I / c) B_{i} \mathcal{L}_{i} \quad F_{m} \approx(I / c) B_{m} \mathcal{L}_{m}$

where $\mathcal{L}_{i}, \mathcal{L}_{m}$ are the effective lengths of the respective segments; the current $I$ is the same in both. From conservation of magnetic flux

$B_{i} \mathcal{L}_{i} h_{i} \approx B_{m} \mathcal{L}_{m} h_{m}$

where $h_{i}, h_{m}$ are the effective widths of the segments in the direction transverse to the current. From Eqs. (5) and (6), the ratio of the Lorentz force in the ionosphere to that in the magnetosphere is

$$
\begin{aligned}
F_{i} / F_{m} \approx h_{m} / h_{i} & \approx\left[\left(B_{i} / B_{m}\right)\left(\mathcal{L}_{i} / h_{i}\right)\left(h_{m} / \mathcal{L}_{m}\right)\right]^{1 / 2} \\
& \sim \sqrt{B_{i} / B_{m}}
\end{aligned}
$$

where the last approximation follows from the reasonable assumption that the ratio of width to length does not differ greatly between the two regions.

In the simplest description of tangential drag at the magnetopause, the sunward Lorentz force of the magnetospheric segment $\mathcal{M}-\mathcal{M}^{\prime}$ is balanced by deceleration of plasma flow in the magnetosheath and thus extracts antisunward linear momentum from the solar wind. The associated antisunward Lorentz force of the ionospheric segment $\mathcal{I}-\mathcal{I}^{\prime}$, balanced by frictional force between plasma and neutrals, imparts antisunward linear momentum to the neutral atmosphere. The amount of momentum imparted here is, however, much larger than the amount extracted from the solar wind: according to Eq. (7), the approximate amplification factor of the ionospheric over the magnetospheric force is inversely proportional to the linear width of the current, hence (by flux conservation) directly proportional to the square root 


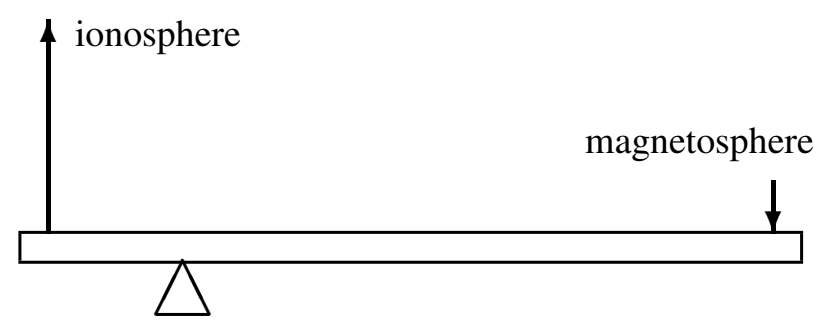

Fig. 2. Relation between forces in ionosphere and magnetosphere for system of region 1 currents (Fig. 1), illustrated by analogy to a simple lever system with mechanical advantage (antisunward is up).

of magnetic field strength - typically a factor $\sim 20$ to 40 . Siscoe and Siebert (2006) reached the same conclusion by carrying out a global MHD simulation of the magnetosphere subject to a strong tangential drag: finding directly that the momentum transfer to the Earth was much larger than that across the magnetopause, they interpreted the difference by a geometrical argument identical to that given here in deriving Eq. (7).

The geometrical argument is in fact quite general and is not restricted to the region 1 current system or direct solarwind interaction. (For example, Fig. 1 with the sense of all the currents reversed can be viewed as a sketch of the region 2 currents (Iijima and Potemra, 1976), the only other difference being that the closure current $\mathcal{M}-\mathcal{M}^{\prime}$ in this case is generally thought to be located in the plasma sheet where the ambient magnetic field is upward, hence the Lorentz force on both the magnetospheric and the ionospheric segments is now sunward.) For any system involving currents that circulate between the magnetosphere and the ionosphere, the Lorentz force exerted by the ionospheric portion is proportional to that exerted by the magnetospheric portion but is also amplified relative to it by a factor, given in Eq. (7), which depends only on the geometrical configuration of the current and of the magnetic field. The situation is analogous to that of a mechanical force amplified by a simple machine, as illustrated in Fig. 2 which represents the Lorentz forces of the configuration in Fig. 1 as forces acting on a lever. The amplification factor is often referred to as the mechanical advantage of the machine.

A further property shared by simple machines and by Lorentz forces in the magnetosphere-ionosphere system is that whereas force is amplified, energy is not. A suitable lever may greatly reduce the force one must apply to raise a given weight, but it does not change at all the amount of work one must do in raising it. Similarly, the rate of electromagnetic energy conversion is given by current times potential difference; the total current is the same in both regions, and as long as the potential difference is the same (analogous to the requirement in the mechanical case that the lever is not being deformed), the rate of energy conversion in the ionosphere is equal in magnitude (and opposite in sign) to that in the magnetosphere.

\section{Implications of amplified Lorentz force}

Given the result (Sect. 2) that the total Lorentz force within a volume must be matched by an equal and opposite Lorentz force somewhere outside the volume, where is this reciprocal force in the case of the Lorentz force on the ionospheric segment of the magnetosphere-ionosphere current system? It cannot be the magnetospheric segment, the Lorentz force on which is smaller by the amplification factor (Sect. 3) and thus cannot be equal (not to mention the possibility that it may even not be opposite, one counterexample being the region 2 current system). A related question is: given the result (Sect. 3) that the linear momentum being imparted to the neutral atmosphere is much larger than that being extracted from the solar wind, where does the additional linear momentum come from?

To the two questions there is a single answer: wherever the reciprocal Lorentz force is located, it extracts linear momentum from the surrounding medium. In terms of the mechanical analog in Fig. 2, this location corresponds to the fulcrum (support) of the lever, the force through the fulcrum being what maintains overall balance with both the direct and the amplified applied force. In the present case, the location evidently must be inward of the ionosphere and hence within the conducting regions of the Earth's interior; furthermore, the existence of the Lorentz force there must be connected with electromagnetic effects of currents in the ionosphere (this is the only way the ionosphere can act across the intervening poorly conducting atmosphere to influence the Earth's interior).

It might be argued that, since the excess Lorentz force is exerted by the Earth's dipole field acting on currents in the ionosphere, it must therefore be balanced by an equal and opposite Lorentz force exerted by the magnetic perturbation fields from the ionosphere acting on the dynamo currents that produce the Earth's dipole. However, since the Lorentz force has a direction fixed relative to the Sun-Earth line, the magnetic perturbation fields from the associated currents necessarily vary with local time; in a frame of reference fixed to the rotating Earth, they appear as time-varying perturbations at the diurnal period and therefore can penetrate into the conducting interior to a depth of no more than about 0.1 or at most $0.2 R_{E}$ (e.g. Price, 1967; Carovillano and Siscoe, 1973), well outside the presumed dynamo region.

The effect of the amplified Lorentz force in the ionosphere on the Earth's interior is thus more complicated than the simple interaction with dynamo currents sketched above; it must involve in an essential way the interior currents that shield out the time-varying external disturbance fields. A proper description requires following the global transfer of linear momentum from the solar wind inward all the way to the center of the Earth. 


\section{Linear momentum transfer from the solar wind}

The total force exerted on the magnetosphere-ionosphereatmosphere-Earth system as the result of interaction with the solar wind can be calculated by integrating the divergence term in Eq. (1) over the volume of the entire system and thus can be expressed as the surface integral of the stress tensor over the outer boundary of the volume. (This surface integral is also, by definition, equal to the rate of linear momentum transfer across the surface.) By separating out the Maxwell stress, the total force can be written as the sum of electromagnetic and mechanical contributions:

$$
\begin{aligned}
\boldsymbol{F}_{E M} & =\int d \boldsymbol{S} \cdot\left[\boldsymbol{B} \boldsymbol{B} / 4 \pi-\mathbf{I}\left(B^{2} / 8 \pi\right)\right] \\
\boldsymbol{F}_{\text {mech }} & =-\int d \boldsymbol{S} \cdot(\rho \boldsymbol{V} \boldsymbol{V}+\mathbf{P}) .
\end{aligned}
$$

Gravity is represented by a term in the total stress tensor $\mathbf{T}$ (e.g., Siscoe, 1970, 1983) but may alternatively and more conveniently be taken into account by adding the term $\rho g$ to the right-hand side of Eq. (1), where $g$ is the gravitational acceleration. Conservation of linear momentum gives

$$
\boldsymbol{F}_{\text {mech }}+\boldsymbol{F}_{E M}+M \boldsymbol{g}=(d / d t) \int d^{3} r \rho \boldsymbol{V}
$$

where

$M=\int d^{3} r \rho$

is the total mass contained within the volume, and $\boldsymbol{g}$ here is only the external gravitational acceleration, due predominantly to the gravity of the Sun (hence $\boldsymbol{g}$ is, to a very good approximation, constant over the volume) - there cannot be any net force or acceleration of the entire Earth system by the gravity of its own internal mass.

As shown in Appendix A, the right-hand side of Eq. (10) can be written as simply $M$ times the acceleration of the center of mass $\boldsymbol{R}$, plus a small correction term. Equation (10) now becomes

$$
\boldsymbol{F}_{\text {mech }}+\boldsymbol{F}_{E M}-\delta \boldsymbol{F}_{m}=M\left(d^{2} \boldsymbol{R} / d t^{2}-\boldsymbol{g}\right)
$$

where

$$
\begin{aligned}
\delta \boldsymbol{F}_{m} & =2(d M / d t)(d \boldsymbol{R} / d t)+\int d \boldsymbol{S} \cdot(\partial \rho \boldsymbol{V} / \partial t)(\boldsymbol{r}-\boldsymbol{R}) \\
& =\int d \boldsymbol{S} \cdot[(\partial \rho \boldsymbol{V} / \partial t)(\boldsymbol{r}-\boldsymbol{R})-2 \rho \boldsymbol{V}(d M / d t)]
\end{aligned}
$$

is a surface integral involving plasma flow and hence can be treated as a correction term to the mechanical force. Note that $\delta \boldsymbol{F}_{m}$ is zero if there is no mass flow across the boundary and also vanishes in a steady state or upon time averaging.

Equation (12) can be applied to any chosen volume, and it is instructive to consider a family of volumes obtained by sequentially displacing the boundary inward: first, the boundary outside the magnetopause, enclosing the entire system;
Table 1. Masses in the Earth-ionosphere-magnetosphere system.

\begin{tabular}{lrr}
\hline Earth & $6 \times 10^{27} \mathrm{~g}$ & $1 M_{E}$ \\
Above surface of Earth $^{\mathrm{a}}$ & $5.2 \times 10^{21} \mathrm{~g}$ & $1 \times 10^{-6} M_{E}$ \\
${\text { Above altitude } 100 \mathrm{~km}^{\mathrm{a}}}^{1.6 \times 10^{15} \mathrm{~g}}$ & $3 \times 10^{-13} M_{E}$ \\
Within magnetosphere $^{\mathrm{b}}$ & $<2 \times 10^{7} \mathrm{~g}$ & $<3 \times 10^{-21} M_{E}$ \\
\hline
\end{tabular}

${ }^{\mathrm{a} C}$ Computed from atmospheric pressure $\simeq$ weight of overlying mass per unit area.

${ }^{\mathrm{b}}$ Rough upper limit, estimated as mass of solar wind plasma (proton concentration $n_{s w}=10 \mathrm{~cm}^{-3}$ ) contained within sphere of radius $10 R_{E}$.

then the boundary just inside the magnetopause; then just above the ionosphere; then just below the ionosphere; then moving by steps into the interior of the Earth, finally converging on the center. For each one of the volumes, the total force on it is determined entirely by quantities on the boundary surface only (left-hand side of the equation) and is equal to the net acceleration - inertial minus gravitational - of the total mass $M$ contained within the entire volume (right-hand side). Since the masses in the atmosphere, ionosphere, and magnetosphere are completely negligible in comparison to the mass of the Earth $M_{E}$ (Table 1), to an extremely good approximation $M$ is equal to $M_{E}$ for all those volumes that include the entire Earth, and the center of mass $\boldsymbol{R}$ coincides with the center of the Earth for all the volumes. The net acceleration $\left(d^{2} \boldsymbol{R} / d t^{2}-\boldsymbol{g}\right)$ has for all practical purposes the same value for all the volumes under consideration; Eq. (12) then implies that the total force on any one volume varies only in proportion to the total mass enclosed.

The total force on the magnetosphere alone (the volume between the magnetopause and the ionosphere) can be calculated by subtracting the forces on two volumes, with boundaries just inside the magnetopause and just above the ionosphere, respectively. The ratio of the resulting net force on the magnetosphere to the (solar-wind-related) force on the Earth is equal to the ratio of the mass in the magnetosphere to the mass of the Earth and hence is extremely small; this is the quantitative reason for the statement (previously derived only qualitatively, Siscoe, 1966; Carovillano and Siscoe, 1973) that the entire force from the solar wind interaction must be transferred to the solid Earth, with negligible net force on the magnetosphere. By the same argument, this holds for other regions (e.g., the ionosphere or even the entire atmosphere), as long as the mass there is much smaller than $M_{E}$.

In Fig. 3, the force (represented by the length of a horizontal arrow) on a particular volume is plotted against a measure of the distance (represented qualitatively by the vertical displacement) between the boundary surface of the volume and the center of the Earth. The horizontal distance between the thin vertical line (zero force) and the dotted line represents 


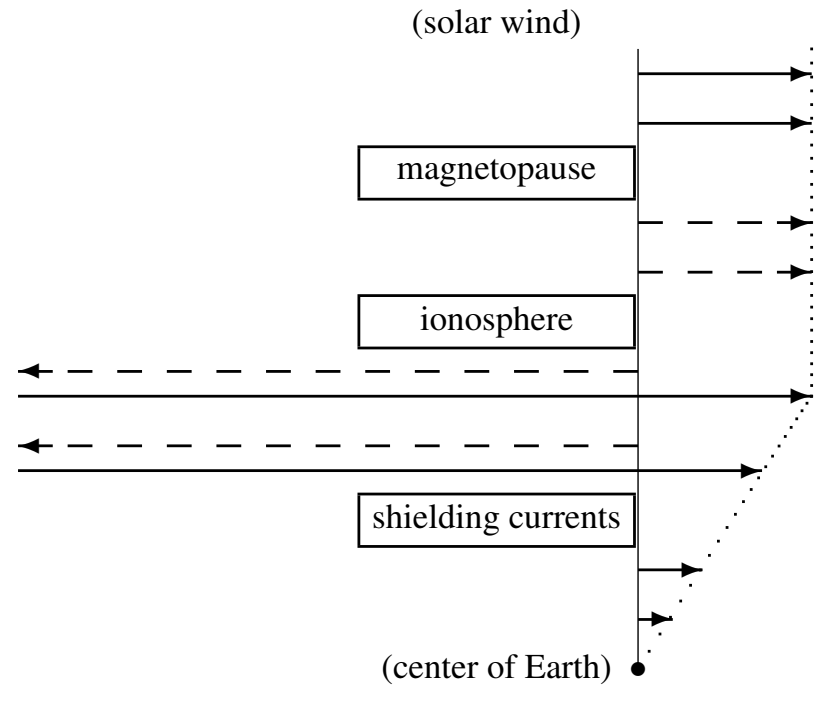

Fig. 3. Total force, calculated from integral of stress tensor over surfaces enclosing varying subvolumes of the Earth-magnetosphere system, separated into mechanical (solid line) and electromagnetic (dashed line) contributions. Arrow to the left (right): force sunward (antisunward). The force at each location acts on the enclosed volume, from the location shown down to the center of Earth.

the total force, without distinction between mechanical and electromagnetic; as discussed above, this force remains constant when the boundary surface is displaced inward from the magnetopause, beginning to decrease (in proportion to the enclosed mass) only when the boundary has been taken below the surface of the Earth.

\subsection{Linear momentum transfer across the magnetopause}

For each assumed boundary surface, mechanical and electromagnetic contributions to the integrated force (or, equivalently, the integrated rate of linear momentum transfer) can be calculated separately, as illustrated in Fig. 3. The stress tensor on the surface just outside the magnetopause is predominantly mechanical, containing both a pressure contribution (magnetosheath plasma pressure, proportional to dynamic pressure of the solar wind), important primarily on the dayside, and a tangential drag contribution (usually ascribed to magnetic tension inside the magnetopause but, as discussed by Vasyliūnas, 1987, describable equally well as flow deceleration stress outside the magnetopause), important primarily in the magnetotail. The stress tensor on the surface just inside the magnetopause is predominantly electromagnetic, magnetic pressure being most important on the dayside and magnetic tension in the magnetotail (Siscoe, 1966; Siscoe and Siebert, 2006; Siscoe, 2006). The total rate of linear momentum transfer is the same on both sides. Within the interaction region just outside and at the magnetopause, the mechanical antisunward force from the flowing solar wind is transformed, by the action of Lorentz force balanced everywhere by appropriate mechanical stresses, into an equal electromagnetic antisunward force just inside the magnetopause; the process is particularly transparent at the boundary of the magnetotail lobes, where antisunward plasma flow is decelerated by sunward tension of open magnetic field lines, leading (as a result of the sharp kink of these field lines) to antisunward tension in the interior region. It is convenient to decompose the force exerted by the solar wind on the entire system into two terms: $F_{s w}=F_{s w 0}+F_{s w 1}$, where $F_{s w 0}$ is associated with currents confined to the magnetopause and $F_{s w 1}$ with currents coupled to the ionosphere (Chapman-Ferraro and region 1 currents, respectively; cf. Siscoe et al., 2002; Siscoe and Siebert, 2006; Siscoe, 2006).

\subsection{Linear momentum transfer through the ionosphere}

For simplicity, I neglect the Lorentz forces within the interior of the magnetosphere compared to those at the magnetopause and in the ionosphere. Then, as illustrated in Fig. 3, the linear momentum transfer across a surface just above the ionosphere is predominantly electromagnetic and its total rate is equal to that across the magnetopause, giving a total force on the enclosed volume that is antisunward and equal to $F_{s w}$. Within the ionosphere itself, however, the Lorentz force exceeds by an amplification factor $A \sim$ at least an order of magnitude (Sect. 3) its counterpart at the nightside interaction region with the solar wind. This implies that the rate of linear momentum transfer across a surface just below the ionosphere must be as shown in Fig. 3: a large sunward electromagnetic force (magnitude $[A-1] F_{s w 1}$ ) balanced by a slightly larger antisunward mechanical force (magnitude $\left.A F_{s w 1}\right)$, the small difference giving, together with the antisunward electromagnetic force $F_{s w 0}$ unaffected by the ionosphere, a net antisunward force on the enclosed volume equal to the force $F_{s w}$ exerted by solar wind, as required.

An equivalent description is that, below the ionosphere, antisunward linear momentum is transported upward by electromagnetic stress and downward by mechanical stress. This arises because the antisunward Lorentz force within the ionosphere (created by the solar-wind interaction but amplified by the mechanical advantage of the magnetosphere) is balanced by collisional friction between plasma and neutral particles; the antisunward linear momentum transferred thereby to the atmosphere is being supplied by an electromagnetic stress and must therefore come from below since there is no adequate source above. At the same time, since there is no net force on the ionosphere-atmosphere system (nor on the magnetosphere above it), the antisunward linear momentum being supplied must be transported downward again by a mechanical stress.

The nature of the mechanical stress that must exist below the ionosphere is not specified by this argument; a daynight pressure gradient as well as a shear stress, e.g., from eddy viscosity, are possibilities. Its magnitude is in any case 
negligibly small in relation to atmospheric dynamics, as can be seen from Table 2 (noting that in order of magnitude $F_{s w}$ should not exceed solar wind dynamic pressure multiplied by the projected cross-section of the magnetosphere).

Ionospheric currents have a horizontal extent that is in general large compared to their vertical extent, and hence they may be treated as thin current sheets, applying methods developed primarily in magnetospheric contexts (e.g. Vasyliūnas, 1983, and references therein). The magnetic disturbance fields on the two sides of a thin current sheet are usually taken to be equal and opposite, so how can the Maxwell stress tensor integrated over a surface just below the ionosphere be larger by an order of magnitude in comparison to that integrated over a surface just above, as required by the preceding discussion? To resolve this apparent puzzle, note that the magnetic field of a thin current sheet can be expressed quite generally as the sum of two terms: the "planar" field, equal and opposite on the two sides, plus the "curvature" field, the same on both sides (terminology introduced by Mead and Beard, 1964). The Maxwell stress tensor is quadratic in the magnetic field, and the only significant contribution to the integral over a closed surface above or below the ionosphere comes from the cross terms, \{dipole $\times$ disturbance field\}. (The \{dipole $\times$ dipole $\}$ terms obviously integrate to zero because the dipole cannot exert a net force on itself; \{disturbance $\times$ disturbance \} could in principle give a nonzero integral when the currents do not close within the ionosphere, but it is of second order in the disturbance field amplitude.) Correspondingly, the electromagnetic force can be expressed as the sum of two sets of terms, \{dipole $\times$ planar and \{dipole $\times$ curvature , giving for the surfaces above and below the ionosphere

$$
\begin{aligned}
& \boldsymbol{F}_{E M}(\text { above })=+\boldsymbol{F}_{E M}(\text { planar })+\boldsymbol{F}_{E M}(\text { curvature }) \\
& \boldsymbol{F}_{E M}(\text { below })=-\boldsymbol{F}_{E M}(\text { planar })+\boldsymbol{F}_{E M}(\text { curvature }) ;
\end{aligned}
$$

the requirements

$$
\begin{aligned}
& \boldsymbol{F}_{E M}(\text { above })= \\
& \boldsymbol{F}_{E M}(\text { below })=(-A+1) \boldsymbol{F}_{s w 1}
\end{aligned}
$$

are then satisfied if

$$
\begin{array}{ll}
\boldsymbol{F}_{E M}(\text { planar }) & =\quad A \boldsymbol{F}_{s w 1} / 2 \\
\boldsymbol{F}_{E M}(\text { curvature }) & =(-A+2) \boldsymbol{F}_{s w 1} / 2 .
\end{array}
$$

The large difference between $\mid \boldsymbol{F}_{E M}$ (above) $\mid$ and $\mid \boldsymbol{F}_{E M}$ (below) $\mid$ arises because the planar and the curvature contributions are of comparable magnitude and thus nearly cancel on one side. The Lorentz force on the ionosphere itself, given by

$$
\begin{aligned}
\boldsymbol{F}_{E M}(\text { above })-\boldsymbol{F}_{E M}(\text { below }) & =2 \boldsymbol{F}_{E M}(\text { planar }) \\
& =A \boldsymbol{F}_{s w 1},
\end{aligned}
$$

has the expected (amplified) value and antisunward direction.
Table 2. Pressures in the Earth-ionosphere-magnetosphere system.

\begin{tabular}{lr}
\hline Solar wind dynamic pressure $^{\mathrm{a}}$ & $2.6 \times 10^{-9} \mathrm{~Pa}^{\mathrm{b}}$ \\
Solar radiation pressure at Earth & $4.7 \times 10^{-6} \mathrm{~Pa}$ \\
Atmospheric pressure at altitude $100 \mathrm{~km}$ & $3.0 \times 10^{-2} \mathrm{~Pa}$ \\
Atmospheric pressure at surface of Earth & $1.0 \times 10^{5} \mathrm{~Pa}$
\end{tabular}

${ }^{\mathrm{a}}$ For $n_{s w}=10 \mathrm{~cm}^{-3}, V_{s w}=400 \mathrm{~km} \mathrm{~s}^{-1}$

$\mathrm{b}_{1} \mathrm{~Pa}=1 \mathrm{~N} \mathrm{~m}^{-2}=10$ dyne $\mathrm{cm}^{-2}$

\subsection{Linear momentum transfer within the Earth's interior}

As long as no non-negligible electric current is crossed while the bounding surface is displaced through the atmosphere and into the Earth's interior, the total electromagnetic force on the enclosed volume remains unchanged: sunward, equal to $[A-1] F_{s w 1}-F_{s w 0}$, and balanced by an antisunward mechanical force that slightly exceeds it in magnitude; the total, net antisunward force no longer equals $F_{s w}$, however, but continually decreases as the enclosed mass becomes significantly less than $M_{E}$. Within the electrically conducting interior, the diurnally varying perturbations of the external magnetic field induce currents that shield out the external field; once the bounding surface has been displaced inward of the layer that contains the shielding currents, there is of course no electromagnetic force of external origin, and the mechanical force is simply equal to the net acceleration multiplied by the remaining enclosed mass. The integrated Lorentz force on the shielding currents, directed sunward, is the equal and opposite reciprocal force (in the sense discussed in Sects. 2 and 4) to the Lorentz force in the ionosphere; it is opposed by an antisunward mechanical stress, of which a small part is associated with the acceleration of the entire Earth system by the net force exerted on it by the solar wind, and the rest - in balance with the Lorentz force - is the source of the additional linear momentum transported to the ionosphere.

It has been tacitly assumed in the preceding discussion as well as in Fig. 3 that $F_{s w 0}$ is small in comparison to $[A-1] F_{s w 1}$, i.e., the electromagnetic force (amplified in the ionosphere) of the region 1 currents greatly exceeds that of the Chapman-Ferraro currents (in the terminology of Siscoe and Siebert, 2006, the thermospheric drag mode dominates over the dipole interaction mode). It is of interest to also consider briefly the opposite limit, the predominantly ChapmanFerraro case (originally described by Siscoe, 1966) with negligible effect of region 1 currents. As illustrated in Fig. 4, the electromagnetic transfer of linear momentum inside the magnetosphere, at the rate corresponding to an antisunward force $F_{s w 0}$ on the enclosed volume, continues essentially unchanged through the ionosphere and into the Earth's interior, until the region of the shielding currents is reached. The integrated Lorentz force on the shielding currents is now directed antisunward (it constitutes the requisite equal and opposite 


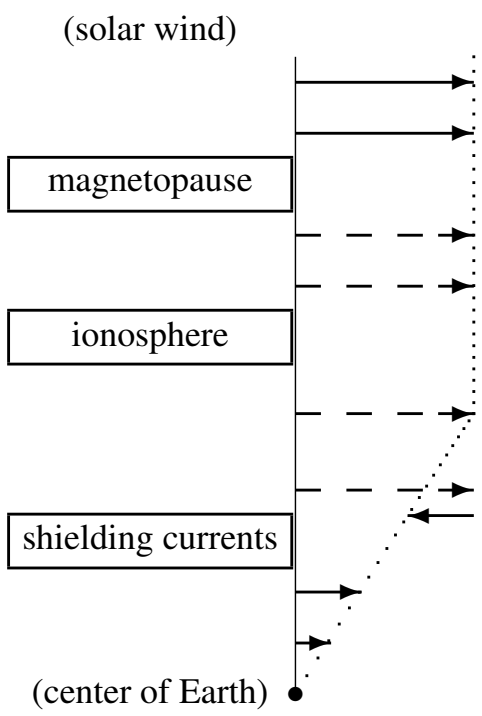

Fig. 4. Same as Fig. 3 but for the case of negligible Lorentz force in the ionosphere.

reciprocal force to the Lorentz force on the Chapman-Ferraro currents), transferring linear momentum to accelerate the enclosed mass. In the region between the shielding currents and the surface of the Earth, where the electromagnetic transport of linear momentum does not change appreciably while the enclosed mass decreases with decreasing radial distance, there is a sunward mechanical force (NB sunward when defined as force on enclosed volume), corresponding to upward transport of antisunward linear momentum in order to enforce the uniform acceleration of the entire Earth.

\subsection{Magnetic gradient force}

The electromagnetic force on the Earth arising from all forms of the interaction with the solar wind has in previous discussions (Siscoe, 1966, 2006; Carovillano and Siscoe, 1973; Siscoe and Siebert, 2006) been described as a force on the Earth's dipole and calculated accordingly from the gradient of the external field at the location of the dipole. In reality, the force is exerted on the shielding currents, the magnetic field of which cancels the external field within the deep interior (including the location of the dipole). The total Lorentz force on the shielding current region can be calculated, analogously to the Lorentz force on the ionosphere (Sect. 5.2), by exploiting again the quadratic nature of the Maxwell stress tensor to write the force as the surface integral of the cross terms \{dipole $\times$ disturbance field $\}$, with the disturbance field now expressed as the sum of the external field and the field of the shielding currents. Hence

$$
\begin{aligned}
& \boldsymbol{F}_{E M}(\text { above })=\boldsymbol{F}_{E M}(\text { external })+\boldsymbol{F}_{E M}^{>}(\text {shielding }) \\
& \boldsymbol{F}_{E M}(\text { below })=\boldsymbol{F}_{E M}(\text { external })+\boldsymbol{F}_{E M}^{<}(\text {shielding }),
\end{aligned}
$$

where "above" or "below" now refers to the bounding surface of a volume that includes or does not include, respectively, the shielding current region. Since the external field, by definition, is unaffected by the shielding currents, the term $\boldsymbol{F}_{E M}$ (external) has the same value above or below, given by the standard expression for the force on the dipole exerted by the external field:

$\boldsymbol{F}_{E M}($ external $)=(\boldsymbol{\mu} \cdot \nabla) \boldsymbol{B}_{\text {ext }}(0)$

( $\boldsymbol{\mu}=$ dipole moment, $\boldsymbol{r}=0$ location of dipole).

\{dipole $\times$ shielding-field terms above and below are

$\boldsymbol{F}_{E M}^{>}$(shielding) $=0$

The

$\boldsymbol{F}_{E M}^{<}$(shielding $)=-\boldsymbol{F}_{E M}($ external $)$.

The first line of Eq. (20) can be deduced by noting that the term $\boldsymbol{F}_{E M}^{>}$(shielding), combined with the \{dipole $\times$dipole and $\{$ shielding $\times$ shielding $\}$ terms (both $=0$ ), represents the total Lorentz force on the combined system of dipole (dynamo) and shielding currents, which vanishes by the corollary at the end of Sect. 2; the second line follows, by virtue of Eq. (18), simply from $\boldsymbol{F}_{E M}$ (below)=0, the absence of the electromagnetic force when the external field has been shielded out. The Lorentz force on the shielding current region itself is then given by

$$
\begin{aligned}
\boldsymbol{F}_{E M} \text { (above) } & -\boldsymbol{F}_{E M} \text { (below) } \\
= & \boldsymbol{F}_{E M}^{>}(\text {shielding })-\boldsymbol{F}_{E M}^{<} \text {(shielding) } \\
= & (\boldsymbol{\mu} \cdot \nabla) \boldsymbol{B}_{\text {ext }}(0)
\end{aligned}
$$

and is precisely equal to the force that the external field alone, in the absence of shielding currents, would exert on the dipole. The total force is thus unaffected by the presence of shielding (a result that, depending on one's degree of insight, may or may not be considered obvious), and the answer obtained in previous calculations is correct.

\section{Limits to Fukushima's theorem}

A necessary condition for global force balance as described in Sects. 4 and 5 is the penetration of magnetic disturbance fields associated with region 1 currents into the Earth's interior, down to depths where the diurnally varying external fields are shielded out. This poses a problem because the penetration of these fields below the ionosphere is generally thought to be severely limited by a famous theorem proved by Fukushima (1969), following earlier suggestions by Boström (1964) and Kern (1966) (for a historical account of the origin of the theorem, see Fukushima, 1985a, part I, Sect. 4).

The theorem in its original form, applicable in the approximation of planar ionosphere and vertical magnetic field lines, is illustrated in Fig. 5: a system of Birkeland currents closing through a general configuration of horizontal currents in the ionosphere (A) can be decomposed into a superposition 
of Birkeland currents closing via potential (zero-curl) ionospheric currents (B) plus source-free (zero-divergence) currents confined to the ionosphere $(\mathrm{C})$; the theorem then states that the magnetic disturbance fields of (B) are identically zero below the ionosphere, the contributions from the Birkeland currents being cancelled by those from the potential currents in the ionosphere. The theorem also applies in the case of spherical ionosphere and radial magnetic field lines; this was first mentioned by Fukushima (1971), citing a 1970 conference presentation by Vasyliūnas (which, however, remained unpublished), and was later derived by Fukushima (1985b).

The method used by Fukushima $(1969,1971,1985 b)$ to derive the theorem is in essence a clever deconstruction into geometrical elements sufficiently simple that the result for each one is evident by symmetry. In Appendix B, I present a purely formal mathematical proof, for both planar and spherical geometries. The decomposition, indispensable to the theorem, of the height-integrated horizontal ionospheric current $\boldsymbol{I}$ into potential and source-free components, proposed by Kern (1966) and extensively used by Vasyliūnas (1970), can be expressed mathematically as

$\boldsymbol{I}=\nabla \tau+\hat{\boldsymbol{r}} \times \nabla \psi$

where $\tau(\theta, \phi)$ and $\psi(\theta, \phi)$ are scalar functions; by continuity of current,

$\nabla^{2} \tau=-\boldsymbol{J} \cdot \hat{\boldsymbol{r}} \equiv-J_{r}=-J_{\|} \hat{\boldsymbol{B}} \cdot \hat{\boldsymbol{r}}$

where $J_{\|}$is the Birkeland current density at the top of the ionosphere. In the case of nearly vertical magnetic field at low altitudes and of ionospheric conductances independent of latitude and longitude, the potential and the source-free currents are the height-integrated Pedersen and the heightintegrated Hall current, respectively, with $\tau$ and $\psi$ both proportional to the electric potential multiplied by the appropriate conductance.

Although the Fukushima theorem does allow penetration of some external disturbance fields below the ionosphere, the fields are those from the source-free (Hall) component of ionospheric currents - from precisely the ionospheric currents that do not couple to the magnetosphere and thus do not constitute the ionospheric segment of the region 1 current system. The penetrating disturbance fields allowed by the theorem induce their own Lorentz force in the shielding current region, equal and opposite to the Lorentz force on the source-free currents in the ionosphere; there is no net force on the Earth system, and no direct connection to force balance from solar-wind interaction. Thus, the problem posed by the Fukushima theorem still remains: how is the Lorentz force on the potential (Pedersen) component of the ionospheric currents - the currents that directly couple to the magnetosphere, that according to the theorem produce no ground-level magnetic disturbance - to be matched with the necessary reciprocal Lorentz force?

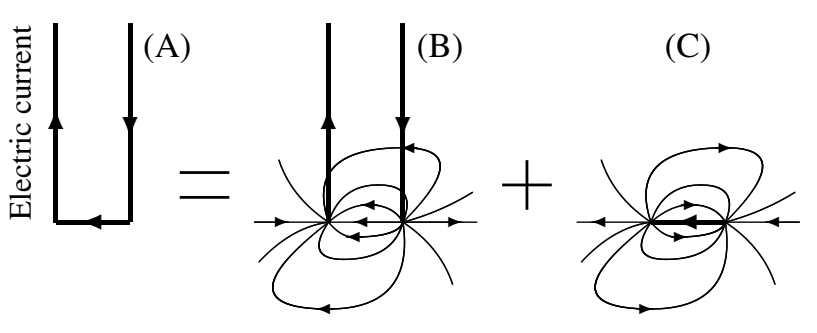

Fig. 5. Simple illustration of Fukushima's theorem (after Fukushima, 1969, 1985b).

As a first step, I calculate the integrated Lorentz force on just the potential part of the ionospheric currents (first term on the right-hand side in Eq. 22), both to verify that it is indeed non-zero and as input to subsequent force balance arguments. The force in the $\hat{\boldsymbol{x}}$ direction is given by the global integral over the ionosphere

$F_{x}=R_{E}^{2} \int d \Omega \hat{\boldsymbol{x}} \cdot \boldsymbol{I} \times \boldsymbol{B}_{d} / c=R_{E}^{2} \int d \Omega \nabla \tau \cdot \boldsymbol{B}_{d} \times \hat{\boldsymbol{x}} / c$

where

$\int d \Omega \equiv \int \sin \theta d \theta \int d \phi$

and $\boldsymbol{B}_{d}$ is the dipole field. With $\tau(\theta, \phi)$ related by Eq. (23) to the radial (Birkeland) currents $J_{r}(\theta, \phi)$, Eq. (24) can be transformed into an integral over $J_{r}$; the calculation is given in Appendix C, with the result

$F_{x}=-\left(B_{E} R_{E}{ }^{3} / 2 c\right) \int d \Omega J_{r} \sin \theta \sin \phi$

$(\theta, \phi$ in geomagnetic coordinates, with signs appropriate for Earth; $B_{E}$ is the surface field strength at the equator). A simple model for region 1 Birkeland currents (basis for many analytical calculations of magnetospheric convection, e.g., Vasyliūnas, 1970, 1972) is

$J_{r}=J_{0} \sin \phi\left[\delta\left(\theta-\theta_{p c}\right)+\delta\left(\theta-\pi+\theta_{p c}\right)\right]$

where $\theta_{p c}$ is the colatitude of the polar cap, and the parameter $J_{0}$ is related to the total current $I_{o}$ of the region 1 system by

$I_{0}=4 J_{0} R_{E}^{2} \sin \theta_{p c}$.

Inserting Eq. (26) into Eq. (25) gives the force at once as

$F_{x}=-\left(\pi B_{E} I_{0} R_{E} / 4 c\right) \sin \theta_{p c} \equiv-F_{T}$,

the minus sign indicating that the force is antisunward.

Equation (28) is the total Lorentz force, integrated over the entire ionosphere. It is also possible to calculate partial Lorentz forces, specifically the force on the polar caps only and the complementary force on the low-latitude region (excluding the polar caps). This calculation (Appendix C) requires the explicit solution of Eq. (23) for $\tau$, given $J_{r}$; with 
$J_{r}$ assumed specified by Eq. (26), the results are

$$
\begin{aligned}
& F_{x}(\text { polar cap })=F_{T}\left\{-2+\left[\sin \theta_{p c} /\left(1+\cos \theta_{p c}\right)\right]^{2}\right\} \\
& F_{x}(\text { low-lat })=F_{T}\left\{+1-\left[\sin \theta_{p c} /\left(1+\cos \theta_{p c}\right)\right]^{2}\right\}
\end{aligned}
$$

the sum of the two giving, as expected, $F_{x}=-F_{T}$ in agreement with Eq. (28).

The results in Eqs. (28), (29), and (30) differ from those of Siscoe (2006), who also assumed the Birkeland current configuration of Eq. (26) but treated the ionosphere in the planar approximation (formally, the limit $\sin ^{2} \theta_{p c} \ll 1$ ), obtaining (in my notation): $F_{x}($ polar cap $)=-2 F_{T}, F_{x}($ low-lat $)=0$, hence $F_{x}$ (total) $=-2 F_{T}$. In Appendix D, I show that the flat-Earth approximation is the reason for these discrepancies as well as some other paradoxical features.

\subsection{Force balance in the idealized geometries}

There are two idealized geometries for which Fukushimas's theorem holds exactly: planar (straight field lines), and spherical (radial field lines). Although these geometries are of course quite inadequate as models for any known existing magnetosphere, situations can be envisaged where they might constitute a reasonable approximation; the radial-field case in particular has been discussed as the extreme limit of a plasma-dominated magnetosphere (Parker and Stewart, 1967). It is instructive to consider how Fukushima's theorem can be reconciled with force balance in such a case.

In the planar case there is no problem at all: the magnetic field has the same magnitude in the ionosphere as in the magnetosphere, hence there is no amplification of the Lorentz force - the linear momentum being imparted to the neutral atmosphere is equal to that being extracted from the solar wind.

In the radial-field case, the integrated Lorentz force on the ionosphere is given (Appendix C) by

$F_{x}=-\left(B_{E} R_{E}^{3} / c\right) \int d \Omega J_{r} \sin \theta \sin \phi /(1+|\cos \theta|)(31)$

different slightly but not significantly from $F_{x}$ in Eq. (25), and of course greatly amplified relative to the magnetospheric/solar-wind value. An additional Lorentz force exists, however, in this case: the force exerted by the region 1 disturbance fields $\delta \boldsymbol{B}$ acting on the current sheet in the equatorial plane (separating the oppositely directed radial fields of the two hemispheres). With the radial magnetic field of Eq. (C18), the current density is $\boldsymbol{J}=\boldsymbol{I} \delta(z)$ where

$(4 \pi / c) \boldsymbol{I}=-2 B_{E} \hat{\boldsymbol{\phi}}\left(R_{E} / r\right)^{2}$,

and the Lorentz force is given by the integral over the current sheet in the equatorial plane

$F_{x(e q)}=\oint d \phi \int_{R_{E}} r d r \hat{\boldsymbol{x}} \cdot \boldsymbol{I} \times \delta \boldsymbol{B} / c$.
From Eqs. (B1) and (B13),

$\delta \boldsymbol{B}=\nabla \alpha \times \hat{\boldsymbol{r}}$,

comparison of Eq. (B14) at $r=R_{E}$ with Eq. (22) establishing that $\alpha=(4 \pi / c) \tau$; then

$$
F_{x(e q)}=\left(2 B_{E} / c\right) \oint d \phi \int_{R_{E}}\left(R_{E} / r\right)^{2} d r \hat{\boldsymbol{x}} \cdot \hat{\boldsymbol{r}} \partial \tau /\left.\partial \phi\right|_{\theta=\frac{\pi}{2}}
$$

which can be rewritten, by carrying out the integration over $r$ and integrating by parts over $\phi$, as

$F_{x(e q)}=+\left(2 B_{E} R_{E} / c\right) \oint d \phi \tau\left(\theta=\frac{\pi}{2}, \phi\right) \sin \phi$

(the upper limit of the $r$-integration has been taken nominally as $\infty$ ). Equation (36) for the Lorentz force integrated over the equatorial current sheet is identical, except for the opposite sign, with Eq. (C22) for the Lorentz force integrated over the ionosphere (for the case of radial field lines); note that this result is completely general and does not depend on any particular choice (e.g., Eq. 26) of the Birkeland current configuration.

The absence of magnetic perturbations below the ionosphere in the radial-field-line magnetosphere, implied by the exact validity of Fukushima's theorem, is thus possible because the Lorentz force reciprocal to that in the ionosphere is here to be found in the equatorial current sheet; the linear momentum being imparted to the neutral atmosphere is extracted from plasma at the reversal of the radial magnetic field. Antisunward linear momentum is predominantly transported downward, from the current sheet to the ionosphere, by electromagnetic stress, with balancing upward transport by mechanical stress; below the ionosphere there is only a small, purely mechanical stress, needed to exert the force $F_{s w}$ on the Earth.

\subsection{Fukushima's theorem in a realistic geometry}

The pattern of momentum tranfer described in the preceding paragraph, demanded by the assumption of no perturbation fields below the ionosphere, is manifestly implausible for any magnetosphere not dominated by the enormous plasma stresses that would be needed to maintain the limiting radial-field-line configuration of Parker and Stewart (1967). Systematic deviations from Fukushima's theorem are therefore of fundamental importance. They were identified already by Fukushima (1985b) as due to a curvature effect: the theorem holds if currents in and out of the ionosphere flow along radial lines, but the real Birkeland currents flow along the (curved) dipolar field lines. As illustrated in Fig. 6, the real current pattern $(\mathrm{F})$ can be represented as a superposition of currents flowing along dipole field lines but closing radially (F1), plus radial currents closing through the ionosphere (F2). Below the ionosphere, the perturbation field of (F2) vanishes by Fukushima's theorem; the perturbation field of the real system $(\mathrm{F})$ must therefore be equal to that of $(\mathrm{F} 1)$. 
It remains only to verify that system (F1) has the appropriate value of the Lorentz force. The contributions from the dipole segments are zero because $\boldsymbol{J} \times \boldsymbol{B}=0$. To evaluate the contributions from the radial segments, note that on them

$J_{r}=-J_{r}\left(R_{E}, \theta, \phi\right)\left(R_{E} / r\right)^{2}$

where $J_{r}\left(R_{E}, \theta, \phi\right)$ is the current flowing into the ionosphere, and the minus sign appears because the current on the radial segment is opposite to the Birkeland current. Then the integrated Lorentz force is

$F_{x(r)}=-\int d \Omega \int_{R_{E}} r^{2} d r J_{r} \hat{\boldsymbol{x}} \cdot \hat{\boldsymbol{r}} \times \hat{\boldsymbol{\theta}} B_{\theta} / c$.

With $B_{\theta}=-B_{E}\left(R_{E}^{3} / r^{3}\right) \sin \theta$ and $\hat{\boldsymbol{x}} \cdot \hat{\boldsymbol{r}} \times \hat{\boldsymbol{\theta}}=-\sin \phi$, carrying out the integration over $r$ gives

$F_{x(r)}=-\left(B_{E} R_{E}{ }^{3} / 2 c\right) \int d \Omega J_{r} \sin \theta \sin \phi$

which is identical with Eq. (25), including the sign; this again is a general result, independent of any specific model for the Birkeland current configuration. The Lorentz force in the interior is the result of shielding out the perturbation fields which are identical with those of (F1); it can thus be viewed indifferently as the reciprocal force either to the Lorentz force of $(\mathrm{F} 1)$ or to the real Lorentz force on the ionosphere.

\section{Conclusions}

As a consequence of the strongly converging geometry of the geomagnetic field lines combined with the condition of current continuity, the Lorentz force on the ionospheric segment of any current system flowing between the ionoasphere and the magnetosphere is greatly amplified relative to the Lorentz force on the magnetospheric or solar-wind segment; the magnetosphere may be said to possess a mechanical advantage over the ionosphere. The forces arising out of the interaction with the solar wind are thus much larger within the ionosphere-atmosphere-Earth system than within the magnetosphere. The total force on the entire system, however, is limited by the rate of extraction of linear momentum from the flow of the solar wind. The large amplified forces must therefore be primarily internal forces between one part of the system and another. Specifically, the antisunward Lorentz force of the region 1 currents in the ionosphere is balanced by a sunward mechanical reaction force of the thermosphere, which is transferred downward through the atmosphere into the Earth's interior, where it is matched by an antisunward mechanical force balanced by the sunward Lorentz force of the electrical currents that shield out the time-varying external magnetic fields.

The following are some of the principal implications:

1. The mechanical stresses on the neutral medium that result from the interaction of the magnetosphere with the

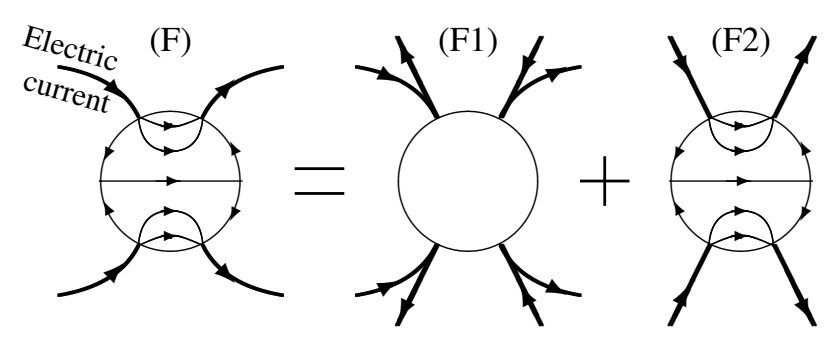

Fig. 6. Curvature effect in Fukushima's theorem (after Fukushima, 1985b).

solar wind are not confined to the thermosphere but extend also into the atmosphere and into that part of the Earth's interior into which any diurnally varying magnetic disturbances can penetrate.

2. The forces involved can be much larger than the net force exerted by the solar wind on the entire Earth system (although they still are utterly negligible compared to with forces involved in dynamics of the atmosphere, let alone of the solid Earth).

3. Except in the case of very weak region 1 currents (in the quantitative sense discussed by Siscoe and Siebert, 2006), the total magnetic force on the Earth's interior is sunward; it is slightly overbalanced by an antisunward mechanical force, the difference being equal to the net force exerted by the solar wind.

4. The magnetic disturbance fields in the Earth's interior that result from the region 1 currents play an essential role in maintain stress balance; hence limits on them imposed by Fukushima's theorem necessarily are of restricted applicability (the requisite deviations from the ideal theorem have been identified already by Fukushima, 1985b).

\section{Appendix A}

\section{Acceleration of center of mass}

Introduce the center of mass $\boldsymbol{R}$ through the definitions

$M \boldsymbol{R} \equiv \int d^{3} r \rho \boldsymbol{r} \quad M \equiv \int d^{3} r \rho$

Noting that $\boldsymbol{r}$ and $t$ are independent variables, we have

$d M / d t=\int d^{3} r \partial \rho / \partial t=-\int d \boldsymbol{S} \cdot \rho \boldsymbol{V}$

and

$d(M \boldsymbol{R}) / d t=\int d^{3} \boldsymbol{r} \boldsymbol{r} \partial \rho / \partial t=\int d^{3} r \rho \boldsymbol{V}-\int d \boldsymbol{S} \cdot \rho \boldsymbol{V} \boldsymbol{r}$ 
where in both equations the second expression follows from the mass continuity equation, plus an integration by parts in Eq. (A3). Differentiating once more with respect to $t$ and rearranging, we have

$$
(d / d t) \int d^{3} r \rho \boldsymbol{V}=d^{2}(M \boldsymbol{R}) / d t^{2}+\int d \boldsymbol{S} \cdot(\partial \rho \boldsymbol{V} / \partial t) \boldsymbol{r}
$$

which can be manipulated into the form

$$
\begin{aligned}
(d / d t) & \int d^{3} r \rho \boldsymbol{V}=M d^{2} \boldsymbol{R} / d t^{2} \\
+ & 2(d M / d t)(d \boldsymbol{R} / d t)+\int d \boldsymbol{S} \cdot(\partial \rho \boldsymbol{V} / \partial t)(\boldsymbol{r}-\boldsymbol{R})
\end{aligned}
$$

from which Eq. (12) follows.

\section{Appendix B}

\section{Formal derivation of Fukushima's theorem}

Represent the disturbance magnetic field (not the total field) by Euler potentials

$\boldsymbol{B}=\nabla \alpha \times \nabla \beta$

then the current density is given by (ignoring the factor $4 \pi / c$ in Gausian or $\mu_{0}$ in SI units)

$$
\nabla \times \boldsymbol{B}=\nabla \alpha \nabla^{2} \beta+(\nabla \beta \cdot \nabla) \nabla \alpha-\nabla \beta \nabla^{2} \alpha-(\nabla \alpha \cdot \nabla) \nabla \beta
$$

For the planar case as in Fig. 5 (vertical direction taken as $\hat{z}$ ), choose

$\alpha=\alpha(x, y) \quad \beta=\beta(z)$

which simplifies Eq. (B2) to

$\nabla \times \boldsymbol{B}=\nabla \alpha \nabla^{2} \beta-\nabla \beta \nabla^{2} \alpha$.

Now require

$\nabla^{2} \beta=d^{2} \beta / d z^{2}=\delta(z)$

$\beta=0 \quad$ for $z<0$

where $\delta(z)$ is merely an idealization of a sharply peaked function; Eqs. (B5) and (B6) are satisfied if

$\nabla \beta=\hat{z} H(z)$

with $H$ the function defined by $H(z)=1 \quad x>0$

$$
H(z)=0 \quad x<0
$$

(Heaviside unit function). From Eq. (B4), the current density is then given by

$\nabla \times \boldsymbol{B}=\delta(z) \nabla \alpha-\hat{\boldsymbol{z}} H(z) \nabla^{2} \alpha$,

which describes a system identical with (B) in Fig. 5: vertical currents in the space $z>0$ (magnetosphere), closed by a curlfree current at $z=0$ (ionosphere). Condition (B6) together with (B1) implies that the disturbance magnetic field of this system vanishes in the space $z<0$ (below the ionosphere), which is Fukushima's theorem.

An analogous calculation can be carried out for the spherical case by choosing $\alpha=\alpha(\theta, \phi), \beta=\beta(r)$. The crossgradient terms in Eq. (B2) are now, unlike the planar case, no longer zero; they can, however, be written as

$(\nabla \beta \cdot \nabla) \nabla \alpha=-(1 / r)(d \beta / d r) \nabla \alpha=-(\nabla \alpha \cdot \nabla) \nabla \beta(\mathrm{B} 9)$

which simplifies Eq. (B2) to

$\nabla \times \boldsymbol{B}=\nabla \alpha\left[\nabla^{2} \beta-(2 / r)(d \beta / d r)\right]-\nabla \beta \nabla^{2} \alpha$.

With the requirements

$\nabla^{2} \beta-(2 / r)(d \beta / d r)=d^{2} \beta / d r^{2}=\delta\left(r-R_{E}\right)$

$\beta=0 \quad$ for $r<R_{E}$

satisfied if

$\nabla \beta=\hat{\boldsymbol{r}} H\left(r-R_{E}\right)$,

the current density is given by

$\nabla \times \boldsymbol{B}=\delta\left(r-R_{E}\right) \nabla \alpha-\hat{\boldsymbol{r}} H\left(r-R_{E}\right) \nabla^{2} \alpha$,

the spherical counterpart of (B) in Fig. 5: a system of radial currents at $r>R_{E}$ closed by a curl-free current at $r=R_{E}$, with vanishing disturbance magnetic field for $r<R_{E}$. (Note: generalizing the theorem even further is not possible, apparently, as the method used here does not seem to work when applied to any other

\section{Appendix C}

\section{Calculation of ionospheric Lorentz force}

Equation (24) may be written as

$F_{x}=R_{E}^{2} \int d \Omega \boldsymbol{Q} \cdot \nabla \tau$

where

$\boldsymbol{Q} \equiv \boldsymbol{B}_{d} \times \hat{\boldsymbol{x}} / c$.

Only the $\hat{\boldsymbol{\theta}}$ and $\hat{\boldsymbol{\phi}}$ components of $\boldsymbol{Q}$ appear in Eq. (C1); they may be separated into curl-free and divergence-free parts by writing

$\boldsymbol{Q}-\hat{\boldsymbol{r}} Q_{r}=(\nabla \mathcal{G}+\hat{\boldsymbol{r}} \times \nabla \mathcal{H}) / r^{2}$

$\left(Q_{r} \equiv \boldsymbol{Q} \cdot \hat{\boldsymbol{r}}\right)$, where $\mathcal{G}(\theta, \phi)$ and $\mathcal{H}(\theta, \phi)$ are scalar functions; $r=R_{E}$ throughout this calculation. Integrating by parts now transforms Eq. (C1) into

$F_{x}=\int d \Omega\left[\nabla \cdot(\mathcal{G} \nabla \tau+\tau \hat{\boldsymbol{r}} \times \nabla \mathcal{H})-\mathcal{G} \nabla^{2} \tau\right] ;$ 
noting that the integral of the divergence over the entire sphere vanishes, we have

$F_{x}=-\int d \Omega \mathcal{G} \nabla^{2} \tau=\int d \Omega \mathcal{G} J_{r}$.

To derive the function $\mathcal{G}$, take the divergence of Eq. (C3):

$$
\left(1 / r^{2}\right) \nabla^{2} \mathcal{G}=\nabla \cdot\left(\boldsymbol{Q}-\hat{\boldsymbol{r}} Q_{r}\right) .
$$

Noting that from Eq. (C2)

$\nabla \cdot \boldsymbol{Q}=\hat{\boldsymbol{x}} \cdot \nabla \times \boldsymbol{B}_{d} / c=0$

and $Q_{r} \sim 1 / r^{3}$, we have

$\nabla_{\Omega}^{2} \mathcal{G}=r Q_{r}=\boldsymbol{r} \cdot \boldsymbol{B}_{d} \times \hat{\boldsymbol{x}} / c=\hat{\boldsymbol{x}} \times \boldsymbol{\mu} \cdot \boldsymbol{r} / r^{3} c \equiv U$.

The Laplacian has been written as $\nabla_{\Omega}^{2}$ to emphasize that $\mathcal{G}$ is a function of $\theta, \phi$ only. The direct solution of Eq. (C8) for $\mathcal{G}$, straightforward but tedious, can be avoided by noting that the expression $U$ is the scalar potential of a dipole field (with moment $\hat{\boldsymbol{x}} \times \boldsymbol{\mu} / c$ ), hence its (3-D) Laplacian is zero, which implies that

$\nabla_{\Omega}^{2} U=-\left(1 / r^{2}\right)(\partial / \partial r) r^{2}(\partial U / \partial r)=-2 U / r^{2}$.

Comparing Eq. (C9) with Eq. (C8), we have

$$
\begin{aligned}
\mathcal{G}=-R_{E}{ }^{2} U / 2 & =-\hat{\boldsymbol{x}} \times \boldsymbol{\mu} \cdot \hat{\boldsymbol{r}} / 2 \\
& =-\left(B_{E} R_{E}{ }^{3} / 2 c\right) \sin \theta \sin \phi,
\end{aligned}
$$

which inserted into Eq. (C5) gives Eq. (28) of the main text.

To calculate the force integrated over only a portion of the entire sphere, the boundary contribution from the divergence term in Eq. (C4) must be included. Specifically, under the assumption (Eq. 26) of Birkeland currents non-zero only at the polar cap boundaries, the forces on the polar-cap and the non-polar-cap regions are given by

$$
\begin{aligned}
\left.F_{x} \text { (polar cap }\right) & =\left(2 / R_{E}^{2}\right) \oint d \phi \mathcal{K}\left(\theta_{p c}^{-}, \phi\right) \\
F_{x}(\text { low-lat }) & =-\left(2 / R_{E}^{2}\right) \oint d \phi \mathcal{K}\left(\theta_{p c}^{+}, \phi\right) \\
\mathcal{K} & \equiv \mathcal{G} \sin \theta(\partial \tau / \partial \theta)-\tau(\partial \mathcal{H} / \partial \phi)
\end{aligned}
$$

(the factor 2 comes from including both hemispheres). The function $\mathcal{H}$ can be derived from Eqs. (C3) and (C10), with use of the relations $\nabla(\boldsymbol{a} \cdot \boldsymbol{r})=\boldsymbol{a}$ for any constant $\boldsymbol{a}$ and $\boldsymbol{Q}-\hat{\boldsymbol{r}} Q_{r}=\hat{\boldsymbol{r}} \times(\boldsymbol{Q} \times \hat{\boldsymbol{r}})$ :

$\mathcal{H}=(3 / 2) \boldsymbol{\mu} \cdot \hat{\boldsymbol{r}} \hat{\boldsymbol{x}} \cdot \hat{\boldsymbol{r}}=-3\left(B_{E} R_{E}{ }^{3} / 2 c\right) \sin \theta \cos \theta \cos \phi(\mathrm{C} 13)$

Solving Eq. (23) for $\tau$, with $J_{r}$ given by Eq. (26), we have

$$
\begin{gathered}
\tau=R_{E}{ }^{2} J_{0} \sin \phi \sin \theta /(1+|\cos \theta|) \\
\theta<\theta_{p c} \quad \pi-\theta_{p c}<\theta \\
=R_{E}{ }^{2} J_{0} \sin \phi\left(1-\left|\cos \theta_{p c}\right|\right) / \sin \theta \\
\theta_{p c}<\theta<\pi-\theta_{p c} .
\end{gathered}
$$

Equations (C11-C12) then give

$$
\begin{aligned}
F_{x}(\text { polar cap }) & =-F_{T}\left(1+3 \cos \theta_{p c}\right) /\left(1+\cos \theta_{p c}\right) \\
\left.F_{x} \text { (low-lat }\right) & =-F_{T}\left(-2 \cos \theta_{p c}\right) /\left(1+\cos \theta_{p c}\right) \\
F_{T} & =\left(\pi B_{E} R_{E}^{3} J_{0} / c\right) \sin ^{2} \theta_{p c},
\end{aligned}
$$

equivalent to Eqs. (29-30) of the main text by virtue of Eq. (27) and the identity

$(1-\cos \theta) /(1+\cos \theta)=[\sin \theta /(1+\cos \theta)]^{2}$.

For the case of radial field lines discussed in Sect. 6.1, $\boldsymbol{B}_{d}$ in Eq. (C2) is replaced by

$\boldsymbol{B}=-\hat{\boldsymbol{r}} B_{E} \operatorname{sign}(\cos \theta)$

(the magnetic flux through a hemisphere is the same as for the dipole field with equatorial field strength $B_{E}$ ). Then $Q_{r}=0$ everywhere, but $\nabla \times \boldsymbol{B}$ and hence also $\nabla \cdot \boldsymbol{Q}$ are nonzero at the singular point $\theta=\pi / 2$ (field reversal), with the result that in place of Eq. (C8) we have

$\nabla_{\Omega}^{2} \mathcal{G}=\left(2 B_{E} R_{E} / c\right) \sin \phi \delta(\cos \theta)$

which has the solution

$\mathcal{G}=-\left(B_{E} R_{E}{ }^{3} / c\right) \sin \theta \sin \phi /(1+|\cos \theta|)$,

the only change from Eq. (C10) being the replacement of a factor 2 in the denominator by $(1+|\cos \theta|)$. Instead of inserting this $\mathcal{G}$ into Eq. (C5), however, it is simpler in this case to derive an alternative expression for $F_{x}$, by using a different integration by parts to transform Eq. (C1) into

$F_{x}=-\int d \Omega \tau \nabla^{2} \mathcal{G}$

and then substituting from Eq. (C19) to obtain

$F_{x}=-\left(2 B_{E} R_{E} / c\right) \oint d \phi \tau\left(\theta=\frac{\pi}{2}, \phi\right) \sin \phi$

which can be compared directly with the force calculated in Sect. 6.1.

\section{Appendix D}

\section{The paradoxes of flatland}

The ionospheric region of primary interest in many discussions of the interaction of the solar wind with the magnetosphere is the high-latitude ionosphere. Relatively small in extent when compared to the entire terrestrial globe, and with the geomagnetic field lines within it inclined only slightly from the vertical, this region is often (particularly for the purpose of calculating simple analytical models) treated in the "flat-Earth" approximation - the ionosphere represented as a plane, with the geomagnetic field perpendicular to it. There exists in fact an exact mapping from a sphere to a 
plane which preserves the form of some of the key equations of magnetosphere-ionosphere coupling theory: the (polar) stereographic projection, in which any point $\mathrm{P}$ on the surface of the sphere is projected onto the plane tangent to the north pole by drawing a straight line from the south pole through P. The coordinates $(\theta, \phi)$ on the surface of the sphere are mapped to cylindrical coordinates $(R, \phi)$ on the plane, with $\phi$ unchanged and $R$ related to $\theta$ by

$$
R / R_{E}=2 \sin \theta /(1+\cos \theta) .
$$

Th equator maps to $R=2 R_{E}$ and the south pole to $R \rightarrow \infty$; the flat-Earth approximation near the north pole is obtained in the limit $R \ll R_{E}$ (or equivalently $\sin \theta \ll 1$ ).

The 2-D Laplacian $\nabla_{\Omega}^{2}$ in $(\theta, \phi)$ coordinates transforms into the 2-D Laplacian in $(R, \phi)$ coordinates, multiplied by the factor $[2 /(1+\cos \theta)]^{2}=\left[1+\left(R / 2 R_{E}\right)^{2}\right]^{2}$. The current continuity Eq. (23) is thus unchanged in form, as is Eq. (22). By direct transformation it can be shown that Eqs. (C1-C5) and (C11-C12) remain valid when expressed in $(R, \phi)$ coordinates. The functions $\mathcal{G}, \mathcal{H}$ become in these coordinates

$$
\begin{gathered}
\mathcal{G}=-\left(B_{E} R_{E}{ }^{2} / 2 c\right) R \sin \phi\{1 /(1+X)\} \\
\mathcal{H}=-3\left(B_{E} R_{E}{ }^{2} / 2 c\right) R \cos \phi\left\{(1-X) /(1+X)^{2}\right\} \\
X \equiv\left(R / 2 R_{E}\right)^{2} ;
\end{gathered}
$$

the factors enclosed in \{\} reduce to \pm 1 in the flat-Earth approximation. It is now apparent why calculating a global force correctly is intrinsically impossible in this approximation: to obtain a global force, the integrals must be taken over the entire plane, but as long as the factors in \{\} remain approximated by $\pm 1, \mathcal{G}$ and $\mathcal{H}$ both diverge for large $R / R_{E}$. (By contrast, for calculations relating the ionospheric electric potential to the Birkeland current distribution, departures from the flat-Earth approximation involve changes only of field magnitude and inclination, which remain bounded over the entire plane.)

As noted already in Sect. 6, calculations by Siscoe (2006) in the flat-Earth approximation give the correct value $-2 F_{T}$ (in the limit $\sin ^{2} \theta_{p c} \ll 1$ ) for the integrated Lorentz force on the polar cap, but not on the low-latitude region; the correct value for the latter is $+F_{T}$, whereas Siscoe (2006) obtains zero, finding that the sunward Lorentz force on the Pedersen currents just equatorward of the polar cap is balanced by the force on the return currents farther away. The physical reason for the discrepancy is that the return currents on the sphere occupy a smaller effective area than on the plane and are also subject to a smaller vertical geomagnetic field. The zero value for the flat-Earth calculation is in fact unique to the boundary condition implicitly assumed by Siscoe (2006), $\tau \rightarrow 0$ at $R \rightarrow \infty$. If one takes instead as the boundary condition $\partial \tau / \partial R=0$ at some fixed (large) $R=R_{1}$, which is physically more realistic (it corresponds to the assumption of zero current across $R=R_{1}$, e.g., the equator), one obtains the integrated Lorentz force on the low-latitude region $=-2 F_{T}$ for any value of $R_{1}$ (the physical reason is that the return currents have been enhanced even further by their confinement to $R \leq R_{1}$ ).

Acknowledgements. I am grateful to G. L. Siscoe for useful discussions.

Topical Editor I. A. Daglis thanks A. Richmond and another referee for their help in evaluating this paper.

\section{References}

Boström, R.: A model of the auroral electrojets, J. Geophys. Res., 69, 4983-4999, 1964.

Carovillano, R. L. and Siscoe, G. L.: Energy and momentum theorems in magnetospheric dynamics, Rev. Geophys. Space Phys. 11, 289-353, 1973.

Fukushima, N.: Equivalence in ground magnetic effects of Chapman-Vestine's and Birkeland-Alfvén's electric currentsystems for polar magnetic storms, Rep. Ionos. Space Res. Japan, 23, 219-227, 1969.

Fukushima, N.: Electric current systems for polar substorms and their magnetic effect below and above the ionosphere, Radio Sci., 6, 269-275, 1971.

Fukushima, N.: Contribution to two selected subjects in Geomagnetism: Electric currents in the ionosphere-magnetosphere, and archaeo-secular variation of geomagnetic field, in: Prospect and Retrospect in Studies of Geomagnetic Field Disturbances, edited by: Nishida, A., Kokubun, S., Iijima, T., Kamide, Y., and Tamao, T., 1-18, Geophysical Research Laboratory, University of Tokyo, 1985a.

Fukushima, N.: Ground magnetic effect of field-aligned currents connected with ionospheric currents: Fundamental theorems and their applications, in: Prospect and Retrospect in Studies of Geomagnetic Field Disturbances, edited by: Nishida, A., Kokubun, S., Iijima, T., Kamide, Y., and Tamao, T., 19-39, Geophysical Research Laboratory, University of Tokyo, 1985b.

Iijima, T. and Potemra, T. A.: The amplitude distribution of fieldaligned currents at northern high latitudes observed by Triad, J. Geophys. Res., 81, 2165-2174, 1976.

Kern, J. W.: Analysis of polar magnetic storms, J. Geomag. Geoelectr., 18, 125-131, 1966.

Landau, L. D. and Lifshitz, E. M.: Fluid Mechanics, Pergamon Press, London, 1959.

Mead, G. D. and Beard, D. B.: Shape of the geomagnetic field solar wind boundary, J. Geophys. Res., 69, 1169-1179, 1964.

Parker, E. N. and Stewart, H. A.: Nonlinear inflation of a magnetic dipole, J. Geophys. Res., 72, 5287-5293, 1967.

Price, A. T.: Electromagnetic induction within the Earth, in: Physics of Geomagnetic Phenomena, Vol. 1, edited by: Matsushita, S. and Campbell, W. H., 235-298, Academic Press, New York, 1967.

Rossi, B. and Olbert, S.: Introduction to the Physics of Space, McGraw-Hill, New York, 1970.

Siscoe, G. L.: A unified treatment of magnetospheric dynamics with applications to magnetic storms, Planet. Space Sci., 14, 947-967, 1966.

Siscoe, G. L.: The virial theorem applied to magnetospheric dynamics, J. Geophys. Res., 75, 5340-5350, 1970. 
Siscoe, G. L.: Solar system magnetohydrodynamics, in: SolarTerrestrial Physics, edited by: Carovillano, R. L. and Forbes, J. M., 11-100, D. Reidel Publishing Co., Dordrecht-Holland, 1983.

Siscoe, G. L.: Global force balance of region 1 current system, J. Atmos. Solar-Terr. Phys., 68, 2119-2126, doi:10.1016/j.jastp.2006.09.001, 2006.

Siscoe, G. L., Crooker, N. U., and Siebert, K. D.: Transpolar potential saturation: Roles of region 1 current system and solar wind ram pressure, J. Geophys. Res., 107(A10), 1321, doi:10.1029/2001JA009176, 2002.

Siscoe, G. L. and Siebert, K. D.: Bimodal nature of solar windmagnetosphere-ionosphere-thermosphere coupling, J. Atmos. Solar-Terr. Phys., 68, 911-920, doi:10.1016/j.jastp.2005.11.012, 2006.

Vasyliūnas, V. M.: Mathematical models of magnetospheric convection and its coupling to the ionosphere, in: Particles and Fields in the Magnetosphere, edited by: McCormack, B. M., 6071, D. Reidel Publishing Co., Dordrecht-Holland, 1970.
Vasyliūnas, V. M.: The interrelationship of magnetospheric processes, in: The Earth's Magnetospheric Processes, edited by: McCormack, B. M., 29-38, D. Reidel Publishing Co., Dordrecht-Holland, 1972.

Vasyliūnas, V. M.: Plasma distribution and flow, in: Physics of the Jovian Magnetosphere, edited by: Dessler, A. J., 395-453, Cambridge University Press, N.Y., 1983.

Vasyliūnas, V. M.: Contribution to "Dialog on the relative roles of reconnection and the 'viscous' interaction in providing solarwind energy to the magnetosphere," in: Magnetotail Physics, edited by: Lui, A. T. Y., 411-412, The Johns Hopkins University Press, Baltimore, 1987.

Vasyliūnas, V. M.: A note on current closure, J. Geophys. Res., 104, 25 143-25 144, 1999. 